NASA Technical Memorandum 84327

NASA-TM-84327 19830011450

\title{
Time Scheduling of a Mix of 4D Equipped and Unequipped Aircraft
}

Leonard Tobias

February 1983

\section{LIRRAMY GPPY}

MAR 181983

LANGLEY RESEARCH CENTER

LIBRARY, NASA

HAMPTON, VIRGINIA 


\section{Time Scheduling of a Mix of 4D Equipped and Unequipped Aircraft}

Leonard Tobias, Ames Research Center, Moffett Field, California

\section{N/SN}

National Aeronautics and

Space Administration

Ames Research Center

Moffett Field. California 94035 


\section{SUMMARY}

In planning for a future air-traffic system that is to be extensively automated, it is necessary to confront the transition situation in which some percentage of the traffic must be handled by conventional means. A safe, efficient transition system is needed since initially not all aircraft will be able to respond to a more automated system, and since it is necessary to encourage users of the system to purchase the equipment required for the more automated operation.

The specific problem addressed in this paper is that of time-scheduling a mix of $4 \mathrm{D}$-equipped aircraft (aircraft that can accurately meet a controller-specified time-schedule at selected way points in the terminal area) when operating in conjunction with unequipped aircraft (aircraft that require air-traffic handing by means of standard vectoring techniques). First, a relationship between timeseparation and system capacity is developed. The conditions under which a $\mathrm{mix}$ of traffic can lead to a capacity improvement have previously been derived. The timeseparations are then incorporated into a set of scheduling algorithms which contain the required elements of flexibility needed for terminal-area operation, such as delaying aircraft and changing time-separations. The problem of reducing the size of time-separations allotted for vectored aircraft by means of computer assists to the controller is also addressed.

\section{INTRODUCTION}

The use of a time-based scheduling system in a terminal area when all aircraft are 4D equipped was investigated earlier in a real-time simulation study. Operational procedures and scheduling techniques were developed which resulted in fewer delays and greater capacity for the time-based system when compared with a standard vectoring mode of operation (ref. 1). Furthermore, the results of the study can be regarded as conservative, since the scheduling algorithm used can be improved on significantly with additional computer-assisted scheduling algorithms.

A more difficult problem, however, arises when operating in an environment in which some aircraft are 4D equipped and some require air-traffic controller handling via standard vectoring techniques. The basic difficulty is that 4D involves a separation of aircraft by time, whereas in the conventional vectoring mode the controllers provide distance-separation. Developing techniques to handle both types effectively is a complicated task. A simple, though inefficient, way to handle both types of scheduling techniques is as follows: (1) time-schedule the 4D-equipped aircraft using methods developed earlier; and (2) for each vectored aircraft assign a very large time slot, say $10 \mathrm{~min}$, so that a controller can surely deliver the aircraft to the scheduling point within the allotted slot. The difficulty with this method is that these large time slots can reduce capacity so that operation in the mixed mode is less efficient than operating in a pure vector mode. Thus, a constraint on the mixed mode to be developed is that it must not result in decreased capacity for the total system.

Another constraint is that the advantages achieved by the 4D-equipped aircraft must not be achieved at the expense of the vectored aircraft; that is, vectored aircraft must still be given a reasonable number of vectors, and must not be delayed more than when all aircraft are being vectored. 
It is clear that the scheduling task is complex when subjected to the above constraints; it may not be clear at this point why it is important to be able to operate effectively in a mixed environment. As mentioned earlier, when most or all aircraft operating in a terminal area are 4D equipped, there are significant fuel and capacity advantages. However, the first users of an on-board 4D system will have to operate in a mixed environment, and they will not be encouraged to switch to a 4D mode unless there are immediate economic advantages.

Thus the objectives of this investigation are (1) to determine the capacity for the mixed-traffic environment and (2) to develop operationally efficient scheduling algorithms to handle a mix of 4D-equipped and unequipped aircraft in the terminal area subject to the following constraints: (1) the capacity is not decreased for the mixed mode compared with the vector mode and (2) in the mixed mode, the vectored aircraft must have neither its fuel consumption nor its pilot's workload increased significantly. This study is restricted to the terminal area. For a general discussion of the Federal Aviation Administration (FAA) automation plans including the en route area see references 2 and 3.

The report is structured as follows: First, a method for obtaining timeseparation requirements is discussed, then the time-separations are used to compute capacity. A set of scheduling algorithms is then outlined followed by a discussion of the assists which must be given to unequipped aircraft. Finally, the concepts presented are incorporated into a real-time air-traffic control (ATC) simulation facility.

\section{ESTABLISHING TIME-SEPARATION REQUIREMENTS}

The present ATC system uses vectors to space aircraft so that minimum separation distance rules are not violated. These minimum separation distances can be converted to a minimum separation time.

For example, suppose that a high-speed aircraft and a low-speed aircraft use the same runway. The high-speed aircraft is traveling at 180 knots and, at the outer marker (located $3.11 \mathrm{n}$. mi. from touchdown), begins its deceleration (at $2 \mathrm{ft} / \mathrm{sec}^{2}$ ) to a final speed of 135 knots. Final speed for the low-speed aircraft is 110 knots. The common path length is $5.09 \mathrm{n}$. mi. This information is summarized in figure 1 .

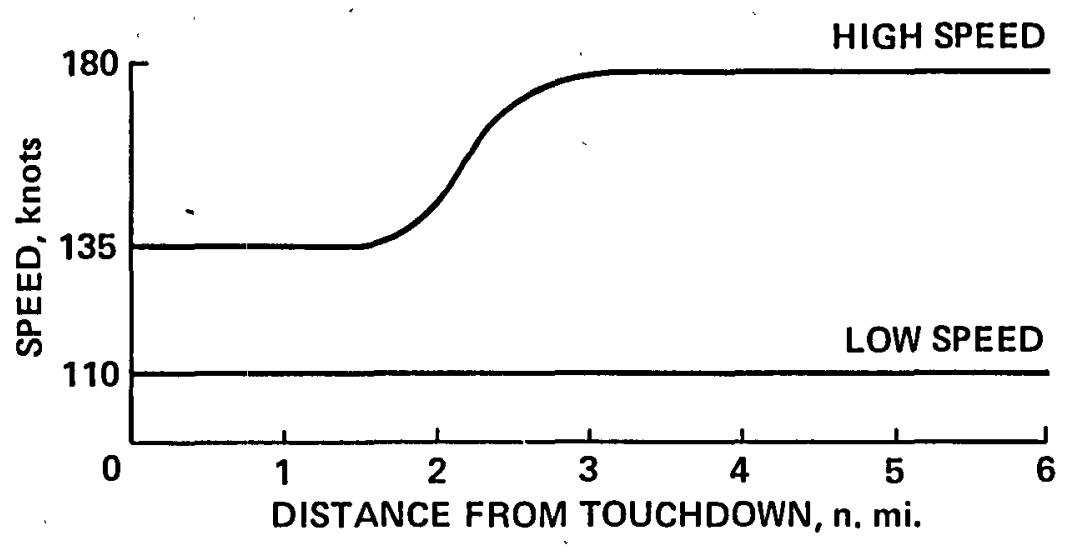

Figure 1.- Sample speed profiles. 
The minimum separation distance $D=\left(d_{i j}\right)$ is given by

$$
D=\left(\begin{array}{ll}
3 & 3 \\
4 & 3
\end{array}\right)
$$

where $d_{i j}$ is minimum separation distance for 1 landing first followed by $j$ ( 1 = low speed; 2 = high speed), $d_{i j}$ in nautical miles.

The corresponding time-separation matrix can be computed to be

$$
\mathscr{T}=\left(\tau_{i j}\right)=\left(\begin{array}{cc}
98 & 74 \\
137 & 74
\end{array}\right)
$$

where $\tau_{i j}$ are in seconds. The elements of this matrix are the time-separations when a lead aircraft is just at touchdown. The times are computed so that the minimum separation distance is not violated for the common path length.

If a separation distance violation occurs in the vicinity of the runway where little or no path-stretching maneuvering is possible, it is necessary to have one of the aircraft execute a missed approach. Thus, in order that missed approaches occur infrequently, a buffer is added by the controller to the minimum spacing. A similar method çan be used to specify the required time-separations in a mixed $4 \mathrm{D}$ environment.

Suppose that an aircraft is to land at $t=t_{a}$. Then the actual touchdown time for a vectored aircraft is assumed to be a normally distributed random variable, $\mathrm{N}\left(\mathrm{t}_{\mathrm{a}}, \sigma_{\mathrm{v}}^{2}\right)$. Similarly, an aircraft that is $4 \mathrm{D}$ equipped is assumed to have a normal distribution, $N\left(t_{a}, \sigma_{4 D}^{2}\right)$. Since the $4 D$-equipped aircraft can achieve tighter time control, $\sigma_{4 D}^{2}<\sigma_{v}^{2}$. The actual values of $\sigma_{4 D}^{2}$ and $\sigma_{V}^{2}$ will have to be obtained by experimental methods. Assuming these values are known, the separation times between any aircraft pairs can be calculated. Let $\tau_{\min }$ be the separation-time obtained from the minimum separation distance requirement. Let $\alpha$ be the first aircraft to land and $\beta$ the second. Furthermore, let

$\tilde{\mathbf{t}}_{\alpha} \quad$ actual time achieved by $\alpha$

$\tilde{\mathrm{t}}_{\beta} \quad$ actual time achieved by $\beta$

$t_{\alpha} \quad$ time assigned for $\alpha$

$t_{\beta}$. time assigned for $\beta$

Thus

$$
\begin{aligned}
& \tilde{\mathrm{t}}_{\alpha}: \mathrm{N}\left(\mathrm{t}_{\alpha}, \sigma_{\alpha}^{2}\right) \\
& \tilde{\mathrm{t}}_{\beta}: \mathrm{N}\left(\mathrm{t}_{\beta}, \sigma_{\beta}^{2}\right)
\end{aligned}
$$

Hence

$$
\tilde{\tau}_{\alpha \beta} \triangleq \tilde{t}_{\beta}-\tilde{t}_{\alpha}: N\left(\tau_{\alpha \beta}, \sigma_{\alpha}^{2}+\sigma_{\beta}^{2}\right)
$$

where $\tau_{\alpha \beta}=t_{\beta}-t_{\alpha}$. 
It is desired to pick $\tau_{\alpha \beta}$, the assigned time-separation, so that

$$
P\left[\tilde{\tau}_{\alpha \beta}<\tau_{m i n}\right]<\mathbf{r}
$$

where $r$ is a small constant, and $P[$ ] is the probability of the event. This is a simple table lookup problem, provided $\sigma_{\alpha}^{2}$ and $\sigma_{\beta}^{2}$ are known. Hence, a set of timeseparations can be determined for each pair of consecutive aircraft.

\section{RELATING TIME-SEPARATION TO CAPACITY}

The ideal capacity with minimum spacing for a first-come, first-served (FCFS) system with no bunching ${ }^{1}$ is given by

$$
C_{\text {ideal }}=\frac{K}{\sum_{i, j} P_{i j} \tau_{i j}} \quad K>0 \text {, constant }
$$

where

$p_{i j}$ probability of aircraft of type $i$. followed by aircraft of type $j$

$\tau_{i j}$ minimum time-separation corresponding to a minimum distance separation for that pair

As described in the previous section, a buffer must be added to these minimum times to prevent separation violations. If $d_{i j}$ is added buffer time, then the actual minimum separation time, $\tau_{i j}^{*}$, is

$$
\tau_{i j}^{*}=\tau_{i j}+d_{i j}
$$

Thus, the actual capacity $\mathrm{C}_{\text {act }}$ is given by

$$
c_{a c t}=\frac{k}{\sum_{i, j} p_{i j}\left(\tau_{i j}+d_{i j}\right)}
$$

and

$$
\frac{c_{\text {ideal }}}{C_{\text {act }}}=1+\frac{\sum_{i, j} p_{i j} d_{i j}}{\sum_{i, j} p_{i j} \tau_{i j}}
$$

${ }^{1}$ For a discussion of capacity under other conditions including arrivals and multiple runways see reference 4 . 
If the arrival rate $\mathrm{N}<\mathrm{C}_{\text {act }}$, then let

$$
N=\frac{K}{\left(\sum_{i, j} p_{i j} \tau_{i j}^{*}\right)+L}, \quad L>0
$$

We wish to allocate $L$ to the $\tau_{i j}^{*}$; thus, let

$$
I=\sum_{i, j} p_{i j} \ell_{i j}
$$

where

$$
\frac{\ell_{1 j}}{L}=\frac{\tau_{i j}^{*}}{\sum p_{i j} \tau_{i j}^{*}}
$$

Now, it is necessary to compare capacities for the mixed modes with the pure 4D or pure vector modes. In the following, all capacities are actual capacities (not ideal), so the asterisk notation will be dropped.

Suppose that a time-separation matrix has been established as follows:

$$
\mathscr{T}=\left(\begin{array}{ll}
\tau_{11} & \tau_{12} \\
\tau_{21} & \tau_{22}
\end{array}\right)
$$

where

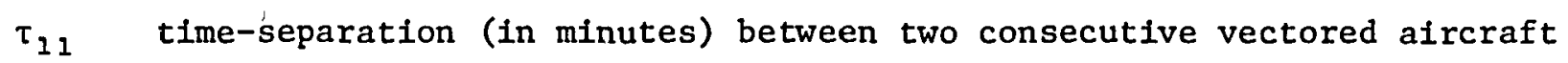

$\tau_{12}$ time-separation for a vectored aircraft followed by a 4D-equipped aircraft

$\tau_{21}$ time-separation for a 4D-equipped aircraft followed by a vectored aircraft

$\tau_{22}$ time-separation between two consecutive 4D-equipped aircraft

Note that the above considers all aircraft to be in the same weight category and to have the same landing speed. In general, the time-separation matrix will thus depend on guidance equipment, weight category, and landing speed.

If all aircraft are vectored, the capacity is given by

$$
\mathrm{C}_{\text {VECTOR }}=\frac{60}{\tau_{11}} \text { aircraft/hr }
$$

and if all aircraft are $4 \mathrm{D}$ equipped, the capacity is

$$
C_{4 D}=\frac{60}{\tau_{22}} \text { aircraft } / \mathrm{hr}
$$


and the relative capacity

$$
\mathrm{R}=\frac{\tau_{11}}{\tau_{22}}
$$

which is assumed to be greater than one.

Let

$$
\tau_{12}=\tau_{22}+\delta, \quad \delta>0
$$

where $\delta$ is defined as the extra time-separation allotted to follow behind a vectored aircraft.

Assume that the time-separation for the unequipped aircraft is the same whether it is following an equipped or unequipped aircraft; thus,

$$
\tau_{11}=\tau_{21}
$$

Let

$a_{E}$ fraction of the aircraft that are 4D equipped

$a_{U} \cdot 1-a_{E}=$ fraction of the aircraft that are vectored

Finally assume that the landing at the runway is first-come, first-served.

Thus, the average interarrival time $I$ is given by

$$
I=\left(\tau_{21} a_{U}+\tau_{22} a_{E}\right) a_{E}+\left(\tau_{11} a_{U}+\tau_{12} a_{E}\right) a_{U}
$$

Substituting (5) and (6) into (7) and simplifying,

$$
I=\tau_{22} a_{E}^{2}+\tau_{11}\left(1-a_{E}^{2}\right)+\delta a_{E}\left(1-a_{E}\right)
$$

The last term in equation (8) can be regarded as a penalty $P$ for mixing 4D-equipped and vectored aircraft; thus,

$$
P=\delta a_{E}\left(1-a_{E}\right)
$$

Note that the maximum penalty occurs when $a_{E}=0.5$, that is, when there is an equal $\mathrm{mix}$ of equipped and unequipped aircraft.

Note further that there is no advantage in capacity if $I \geq \tau_{11}$. This leads to a condition on $\delta$ for the mix capacity to be greater than the capacity of the vectoring mode:

$$
C_{M I X}>C_{V E C T O R} \text { iff } \delta<\frac{a_{E}\left(\tau_{11}-\tau_{22}\right)}{1-a_{E}}
$$


In order to ensure that the mixed mode of operation does not decrease capacity, It may be necessary to provide the controller with computer assists for the vectored aircraft. These would have the effect of narrowing the distribution, that is, reducing the values of $\sigma^{2}$ so that $\sigma_{\mathrm{v}}^{2}>\sigma^{2}>\sigma_{4 D}^{2}$. Depending on just how the assists are provided, they would have the effect of reducing $\tau_{11}$ or $\delta$ or both, thereby reducing the interarrival time and increasing the capacity.

To summarize, then, it is necessary to establish a set of operational procedures and to determine experimentally the time-spacing accuracy that can be achieved by the vector mode, the $4 \mathrm{D}$ mode, and any computer-assisted vector mode. Then, using the techniques described, the time-separation requirements for any aircraft pair must be determined. It is then necessary to develop an efficient set of scheduling algorithms and operational procedures based on these separation requirements.

\section{SCHEDULING ALGORITHM}

In this section, a specific set of scheduling algorithms is discussed. It should be noted, however, that there are no unique solutions; instead, there is a range of' solutions corresponding to any set of specifications and constraints. First, system requirements are discussed, followed by an elaboration of specific scheduling algorithms.

One main function of the air-traffic controller is to monitor his radar screen to detect and prevent conflict situations. If aircraft are to be assigned times and the time interval between aircraft is dependent on a variety of factors, the controller must be provided the scheduling information via a set of algorithms programmed on the ground computer; it is too complex a task to be performed by the controller in addition to the monitoring function. In the previous study, in which all aircraft were $4 \mathrm{D}$ equipped, a system in which the controller would manually schedule a11 aircraft at least $2 \mathrm{~min}$ apart at one schedule point was investigated. This simple scheme was inefficient from a capacity standpoint, since it meant that the timeseparation requirement was established for the slowest pair of consecutive aircraft. In addition, even this simple mode of operation required too much decisionmaking and distraction from monitoring the screen. Hence, a computer algorithm is required to develop an effective landing order which is conflict-free and which assigns timeseparation between aircraft that is dependent on aircraft weight category as well as on the presence or absence of $4 \mathrm{D}$ equipment.

In addition to setting up a schedule, algorithms are required to alter the schedule; otherwise, the schedule is too inflexible. Any alteration which the scheduling system could not handle would require the controllers to revert to the system now in use whereby all aircraft would be vectored. There must be a means of handling missed approaches. The controller also needs to change the rate of flow. It may be that he also requires the ability to block out specific time periods from the computer schedule or require that a few aircraft be scheduled in an order that he specifies. These alterations to the computer-generated schedule might lead to short periods of less efficient operations in terms of fuel or system capacity; however, it must be possible to "recover" and resume the efficient computer-generated schedule operation. With the above constraints in mind, a specific set of algorithms will now be discussed.

Consider a schedule of times $T=\left\{t_{i}\right\}, i=1, \ldots ., n$, where $t_{i}=$ time assignment for aircraft $i$ at the runway and a corresponding set of aircraft types $S=\left\{s_{i}\right\}$, where $s_{i}=$ aircraft type for the $i$ th aircraft. Note that type is to 
include weight category as well as the presence or absence of 4D equipment. Furthermore, let $\mathscr{T}=\left\{\tau_{i j}\right\}$, where $\tau_{i j}$ is the minimum separation time required at the runway when an aircraft of type 1 lands first, followed by an aircraft of type $j$. A schedule $T$ is feasible at the runway if

$$
t_{1+1} \geq t_{1}+\tau_{s_{1}, s_{1+1}}, \quad i=1, \ldots ., n-1
$$

and is sald to have no gaps if the equality holds for all $i$. It may be necessary to alter the schedule, and various types of alterations are briefly considered.

Add gaps to the schedule $T$ - Suppose it is desired to add gaps every $\ell$ aircraft of size w. The modified schedule $T^{\prime}=\left\{t_{i}^{i}\right\}$ is

$$
t_{i}^{\prime}=\left\{\begin{array}{cc}
t_{i} & i=1, \ldots ., l \\
t_{i}+w & i=\ell+1, \ldots . ., 2 \ell \\
\vdots & \vdots \\
t_{i}+k w & i=k \ell+1, \ldots,(k+1) l \quad k<0, i \leq n
\end{array}\right.
$$

Schedule an aircraft (type $\sigma$ ) at or after $t *$ without altering schedule TClearly, if $t *$ occurs at or after $t_{n}$ there is no problem; in particular, if $t * \geq t_{n}+\tau_{s_{n}, \sigma}$, then the aircraft can be scheduled at $t *$. If $t_{n} \leq t *<t_{n}+\tau_{s_{n}, \sigma}$, then schedule it at $t_{n}+\tau_{s_{n}, \sigma}$. Also, if. $t *$ is before $t_{1}-\tau_{\sigma, s_{1}}$, then schedule at $t *$. The more complex situation is when $t *$ is in the range $\left(t_{1}-\tau_{\sigma, s_{1}}, t_{n}\right)$. In this case, it is necessary to search for the first available gap of sufficient size to accommodate an aircraft of type $\sigma$. An algorithm to accomplish this is outlined below.

Step a: Let $\bar{i}=\min i$ such that $t_{\bar{I}} \leq t^{*}<t_{\bar{i}+1}$. The interval of interest is shown below:

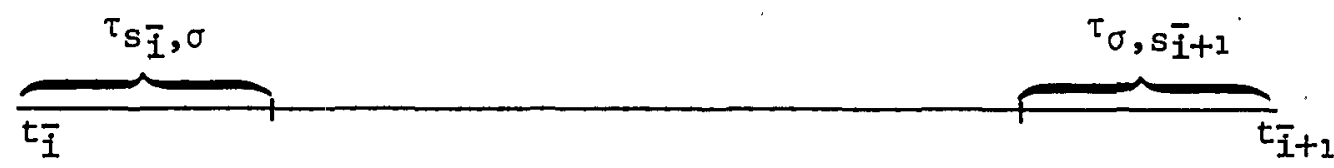

(Note: It is assumed that $t_{\bar{i}+1}-t_{\bar{i}} \geq \tau_{s_{\bar{i}}, \sigma}+\tau_{\sigma, s_{\bar{i}+1}}$. If this is not the case, go . to step b.)

Referring to the figure, it follows that

1. If $t_{\bar{i}} \leq t^{*} \leq t_{\bar{i}}+\tau_{s_{\bar{i}}, \sigma}$, then schedule at $t_{\bar{i}}+\tau_{s \bar{i}, \sigma}$

2. If $t_{\bar{i}}+\tau_{s_{\overline{1}}, \sigma}<t_{*} \leq t_{\bar{i}+1}-\tau_{\sigma, s_{\bar{i}+1}}$, then schedule at $t *$

3. If $t_{\bar{i}+1}-\tau_{\sigma, s \bar{i}+1}<t *<t_{\bar{i}+1}$, go to step $b$

Step $b$ : For $i=\bar{i}+1, \ldots ., n-1$, if $t_{1+1}-t_{i} \geq \tau_{s_{i}, \sigma}+\tau_{\sigma, s_{1+1}}$, schedule at $t_{i}+\tau_{s_{i}, \sigma}$. If $t_{i+1}-t_{i}<\tau_{s_{i}, \sigma}+\tau_{\sigma, s_{i+1}}$ for all $i=\bar{i}+1, \ldots . ., n-1$, schedule at $t_{n}+\tau_{s_{n}, \sigma}$. 
Schedule an aircraft (type $\sigma$ ) at $t^{*}$ by modifying schedule $I-$ Let $\bar{I}$ be such that $t_{\bar{i}} \leq t^{*}<t_{\bar{i}+1}$.

1. If $t^{*} \geq t_{\bar{i}}+\tau_{s_{\bar{i}}, \sigma}$, then aircraft $\bar{i}$ need not be rescheduled. In addition, If it is also true that $t^{*} \leq t_{\bar{i}+1}-\tau_{\sigma, s} \overline{i+j}$, then no aircraft need be rescheduled. If $t *>t_{\bar{i}+1}-\tau_{\sigma, s} \bar{I}+1_{1}$, then $t_{\bar{i}+1}=t *+\tau_{\sigma, s_{\bar{i}+1}}$ and for $1=\bar{i}+2, \ldots, \ldots, n$,

$$
t_{i}^{\prime}=\max \left[t_{i}, t_{i-1}^{\prime}+\tau s_{i-1, s_{i}}\right]
$$

2. If $t *<t_{\bar{i}}+\tau_{s_{\bar{i}}, \sigma}$, then $t_{\bar{i}}=t *+\tau_{\sigma, s_{\bar{I}}}$ and equation (11) is valid for $i=i+1, \ldots ., n$. Similar techniques can be used to open blocks of time, cancel any aircraft, etc.

So far, the schedule manipulation refers to scheduling at a specific point without regard to route structure. Assume now a set of routes emanating from given feeder-fixes. Let $B=\left\{b_{i}\right\}^{\prime}$ be the set of routes followed by an aircraft landing according to the schedule $T$. Also let $R=\left(r_{s_{i}}, b_{i}\right)=$ transit time from feeder-fix to touchdown, as a function of aircraft type and route. Note first that for any feasible schedule $T$, the corresponding feeder-fix times $F=\left\{f_{i}\right\}$ are given by

$$
f_{i}=t_{i}-r_{s_{i}}, b_{i}
$$

and that because the route distances may be different, in general, $F$ is not timeordered. However, along a particular route, it is necessary to preclude overtakes.

Also, it may be necessary to establish a minimum time-spacing at the feeder-fix between consecutive aircraft. Let $\Delta=\left(\delta_{i j}\right)$ be the matrix of separation times where $\delta_{i j}$ time-spacing at the feeder-fix if type $i$ is directly followed by type $j$.

A schedule $T$ is said to be feasible if

1. It is feasible at the runway

2. For any pair $i, j$ such that $b_{i}=b_{j}, i>j$, then $f_{i} \geq f_{j}+\delta_{s_{i}, s_{j}}$

The following is thus denoted the feasible schedule problem: Given a schedule $T$, find a schedule $T^{\prime}$ that is feasible.

First, transform $T$ to a schedule $\tilde{T}$ that is feasible at the runway. The algorithm for this is

$$
\begin{aligned}
& \tilde{t}_{1}=t_{1} \\
& \tilde{t}_{k}=\max \left[\tilde{t}_{k-1}+\tau_{\left.s_{k-1}, s_{k}, t_{k}\right] \quad k=2, \ldots, n} .\right.
\end{aligned}
$$

Now, revise the schedule $\tilde{T}$ so that there are no separation violations between consecutive aircraft along the same route. Thus, for $i=1, \ldots . ., n-1$, 
1. Find the first $j=1+1, \ldots$, ., such that $b_{j}=b_{1}$. (If $b_{j} \neq b_{1}$ for any $j$, set $i=i+1$ and continue)

2. Suppose that for $j=k, b_{k}=b_{i}$, and check if $f_{k} \geq f_{i}+\delta_{s_{i}, s_{k}}$; if the above is valid, set $i=i+1$ and continue

3. If not, set $f_{k}=f_{i}+\delta_{s_{i}, s_{k}}$ and revise $\tilde{t}_{k}$ :

$$
\tilde{t}_{k}=f_{k}+r_{s_{k}, b_{k}}
$$

With this revision of $\tilde{t}_{k}$, it is possible that the remainder of the schedule is not feasible at the runway. Thus, revise $t_{j}$ for $j=k+1, \ldots, n$ so that the schedule is still feasible at the runway.

The above solution actually obtains the minimum-time-feasible solution for the FCFS case. For any feasible $\bar{T}$ of the original schedule $T$, the following are valid:

$$
\begin{aligned}
& \bar{t}_{1} \geq t_{1} \\
& \bar{t}_{i} \geq \max \left[t_{i}, \bar{t}_{i-1}+\tau_{s_{i-1}, s_{i}}, f_{j}+\delta_{s_{j}, s_{i}}+r_{s_{i}, b_{i}}\right]
\end{aligned}
$$

where $j$ is the aircraft that precedes $i$, along route $b_{i}$. For the algorithm developed, equality is achieved at each iteration step:

$$
\begin{aligned}
& t_{i}^{\prime}=t_{1}^{\prime} \\
& t_{i}^{\prime}=\max \left[t_{i}, t_{i-1}^{\prime}+\tau_{s_{1+1}, s_{i}, f_{j}}+\delta_{s_{j}, s_{i}}+r_{s_{i}, b_{i}}\right]
\end{aligned}
$$

An additional problem to be considered is denoted the halt problem: Given a feasible schedule $T$, suppose that all the alrcraft which have departed the feederfix (denoted active aircraft) are permitted to continue undisturbed, while the remaining aircraft are delayed for $t_{h} \mathrm{sec}$. Reschedule the delayed aircraft without altering the time assignments for the active aircraft.

Suppose there are $k$ active aircraft and $n-k$ delayed aircraft. The active aircraft form a feasible schedule denoted $T_{k}$. The delayed aircraft form a feasible schedule $\mathrm{T}_{\mathrm{n}-\mathrm{k}}$. The problem is that because of the delay and because of the differences in time from feeder-fix to runway, some of the active aircraft times may conflict with the delayed aircraft. Clearly, one solution would be to schedule all the delayed aircraft after the last active aircraft. However, it may be possible to interleave some of the delayed aircraft between active aircraft. The algorithm to accomplish this interleaving without altering the schedule of the active aircraft has been discussed previously.

Using the algorithms described or ones similar to the above, one can develop a basic set of scheduling algorithms which essentially automates the time-assignment procedure, but which provides flexibility for the controller to alter the system if he chooses.

One can possibly improve the system operation further by allowing a limited amount of time slot shifting; that is, instead of allocating slots according to firstcome, first-served at the runway, one could use the constrained position shifting 
method developed by Dear (ref. 5). Dear's method allows aircraft to be shifted from an FCFS position to take advantage of bunching speed classes on a 1imited basis.

\section{ASSISTS FOR THE UNEQUIPPED AIRCRAFT}

The critical element for the success of the mixed operation will be the accuracy with which it will be possible for a vectored aircraft to meet an assigned time. Again, it should be noted that the unequipped aircraft will be vectored and that it will not even be aware of a specific time assignment.

There are several ways to assist the controller in vectoring unequipped aircraft to meet an assigned time. First, the controller is aware of the time schedules of the equipped aircraft. If these aircraft are permitted to fly their 4D profile. undisturbed, the controller can use these aircraft as guideposts for spacing the unequipped aircraft. At some terminal areas where two arrival routes merge to a single route, it is now common practice for the controllers to interleave traffic as follows: On one route, space aircraft $10 \mathrm{n}$. mi. apart. On the other route, interleave aircraft between the equally spaced aircraft. The 4D-equipped aircraft can be similarly used as guideposts. Second, it is possible to have schedule times displayed for the unequipped aircraft to provide a guideline for the controller. Third, under the constraint that the unequipped aircraft will be vectored along a fixed path, it is possible for the ground computer system to generate and display an estimated timeerror for unequipped aircraft. Finally, the ground computer can generate and display to the controller speed or heading vectors to correct time-error estimates. The last two options will require additional research.

\section{AIR-TRAFFIC CONTROL SIMULATION FACILITY}

Some of the scheduling algorithms described have been incorporated into the ATC simulation facility at Ames Research Center. The facility, a real-time simulation facility, is used to study advanced terminal-area operational procedures. A photo of a typical air-traffic control display is shown. In figure 2. The aircraft are shown as triangles, and the block of data next to each aircraft indicates' the aircraft identification, status, altitude, and speed. The table in the upper left-hand corner, denoted the flight data table (FDT), provides schedule information. At the top of the table; the present time is shown in hours, minutes, and seconds.. The first column shows the aircraft identification, such as "S1," the status (STA), and the assigned route (RT). Also shown is the expected time of arrival (ETA) at the runway touchdown in minutes and seconds. Thus, T1 will touch down at 13:44:29. The last column is the delay (DY), where the expected delay at touchdown is in seconds.

Aircraft in the FDT below the dotted line are those which will depart the feederfix within $5 \mathrm{~min}$. However, the controller has halted all feeder-fix departures (indicated by "HALT" in the FDT) so that these aircraft will have to be rescheduled: This was necessitated by the aircraft $\mathrm{S} 1$, which is executing a missed approach. The ETA time shown for $\mathrm{S} 1$ will also have to be revised when $\mathrm{S} 1$ is rescheduled. 

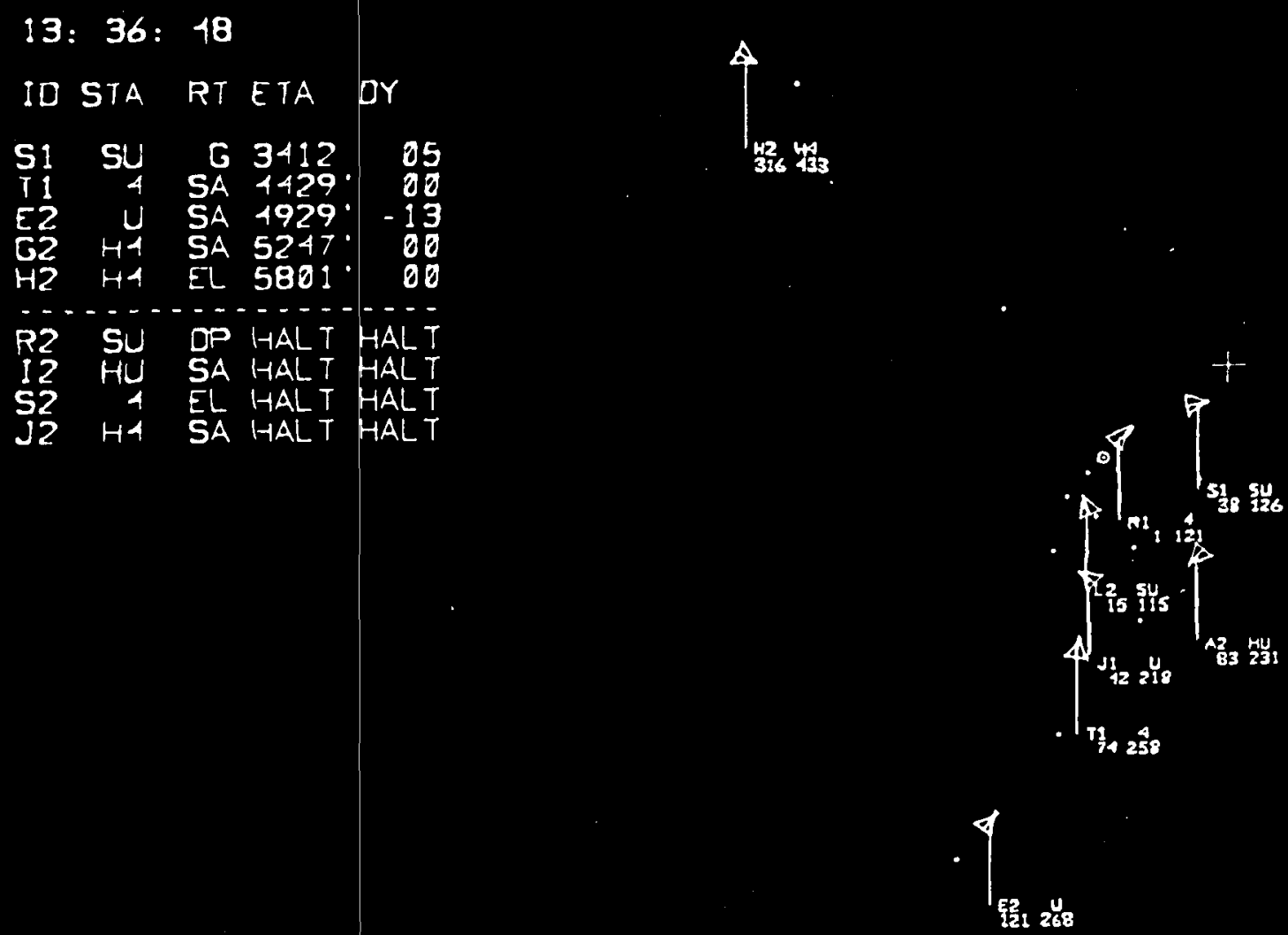

ויויו

Figure 2. - Typical air-traffic control display. 


$$
\text { CONCLUSIONS }
$$

The. use of a time-based ATC system in the terminal area has been analyzed for a $\mathrm{mix}$ of $4 \mathrm{D}$-equipped and unequipped aircraft. The importance of obtaining information on the accuracy of time-scheduling has been shown, and the relationship between timeseparation and system capacity has been developed. In particular, the conditions under which a mix of traffic can lead to a capacity improvement have been derived.

A set of scheduling algorithms has been presented which contains the required elements of flexibility needed for efficient terminal-area operation. It has been shown how an existing schedule can be revised by halting traffic at the feeder-fix and adding gaps to the schedule.

Finally, the topic of computer-assists to the unequipped aircraft, such as providing time information and suggested vectors, has been introduced. This will be investigated in more detail in the real-time simulation facility at Ames Research Center. 
1. Tobias, L.; and O'Brien, P.J.: Real-Time Manned Simulation of Advanced TerminalArea Guidance Concepts for Short Haul Operations. NASA TN D-8499, 1977.

2. Goldmuntz, L.; Kefaliotis, J. T.; Kleiman, L. A.; Rucker, R. A.; Schuchman, L.; and Weathers, D.: The AERA Concept. Federal Aviation Administration, FAA-EM-81-3, Mar. 1981.

3. Alvania, S. M.: Integrated Flow Management. 1982 American Control Conference, Arlington, Va., June 1982.

4. Newe11, G. F.: Airport Capacities and Delays. Transportation Science, vol. 13, no. 3, Aug. 1979, p. 201.

5. Dear, R.: Increasing Capacity with Computer-Assisted Decision Making. Proc. International Air Transportation Conf., vol. 2, May 1979. 


\begin{tabular}{|c|c|c|}
\hline $\begin{array}{l}\text { 1. Report No. } \\
\text { NASA TM } 84327\end{array}$ & 2. Government Accession No. & 3. Recipient's Catalog No. \\
\hline \multirow{2}{*}{\multicolumn{2}{|c|}{$\begin{array}{l}\text { 4. Title and Subtitle } \\
\text { TIME SCHEDULING OF A MIX OF 4D EQUIPPED AND } \\
\text { UNEQUIPPED AIRCRAFT }\end{array}$}} & $\begin{array}{l}\text { 5. Report Date } \\
\text { February } 1983\end{array}$ \\
\hline & & 6. Performing Organization Code \\
\hline \multicolumn{2}{|l|}{$\begin{array}{l}\text { 7. Author(s) } \\
\text { Leonard Tobias }\end{array}$} & $\begin{array}{l}\text { 8. Performing Organization Report No. } \\
\text { A-9225 }\end{array}$ \\
\hline \multirow{3}{*}{\multicolumn{2}{|c|}{$\begin{array}{l}\text { 9. Performing Organization Name and Address } \\
\text { NASA Ames Research Center } \\
\text { Moffett Field, Calif. } 94035\end{array}$}} & $\begin{array}{l}\text { 10. Work Unit No. } \\
\text { T3309 }\end{array}$ \\
\hline & & 11. Contract or Grant No. \\
\hline & & 13. Type of Report and Period Covered \\
\hline \multirow{2}{*}{\multicolumn{2}{|c|}{$\begin{array}{l}\text { 12. Sponsoring Agency Name and Address } \\
\text { National Aeronautics and Space Administration } \\
\text { Washington, D.C. } 20546\end{array}$}} & Technical Memorandum \\
\hline & & $\begin{array}{l}\text { 14. Sponsoring Agency Code } \\
505-34-11\end{array}$ \\
\hline
\end{tabular}

16. Abstract

In planning for a future air-traffic system that is to be extensively automated, it is necessary to confront the transition situation in which some percentage of the traffic must be handled by conventional means. A safe, efficient transition system is needed since initially not all aircraft will be able to respond to a more automated system, and since it is necessary to encourage users of the system to purchase the equipment required for the more automated operation.

The specific problem addressed in this paper is that of time-scheduling a mix of 4D-equipped aircraft (aircraft that can accurately meet a controller-specified time-schedule at selected way points in the terminal area) when operating in conjunction with unequipped aircraft (aircraft that require air-traffic handling by means of standard vectoring techniques). First, a relationship between time-separation and system capacity is developed. The conditions under which a mix of traffic can lead to a capacity improvement have previously been derived. The time-separations are then incorporated into a set of scheduling algorithms which contain the required elements of flexibility needed for terminal-area operation, such as delaying aircraft and changing time-separations. The problem of reducing the size of time-separations allotted for vectored aircraft by means of computer assists to the controller is also addressed.

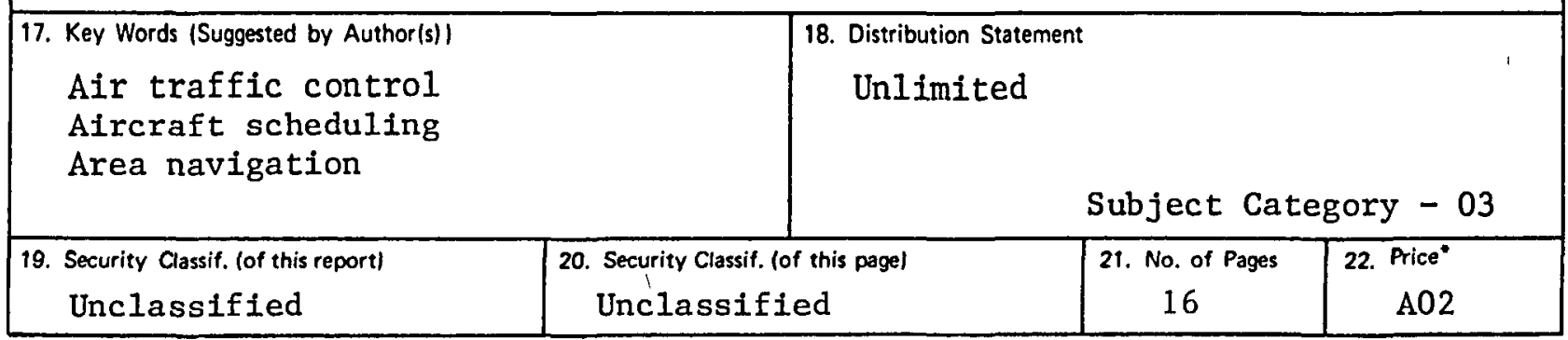

